\title{
Neutron Detector Systems for Neutron Nuclear Data Measurements
}

\author{
Robert C. Haight ${ }^{*}$ \\ Los Alamos Neutron Science Center \\ Los Alamos, NM 87545, USA \\ E-mail: haight@lanl.gov
}

\begin{abstract}
Nuclear data are essential in a wide range of applications including nuclear power reactors, nuclear safety, space radiation effects, medical radiation diagnostics and therapy, radiation effects on semiconductors, passive and active interrogation schemes, radiography, acceleratordriven systems, astrophysics and basic science research. These data are embodied in evaluated data libraries and reaction models and are used in radiation transport codes. The accuracy of the calculations and therefore the credibility of the results depend on the accuracy of the input data. Continuing progress in the quality of these data has led to the present situation where a great variety of effects can be calculated with confidence, with limits being determined often by other considerations such as the representation of the system to be calculated, thermal-hydraulic properties including their time dependence, and so forth. As computer calculations get ever faster and more accurate, however, new demands will be placed on the both on the codes and the input data. Some of the most important data are obtained with neutron detectors. This talk will review the properties of neutron detectors used for nuclear data measurements and the requirements put on them to address the need for more accurate and complete nuclear data. In this review emphasis will be given to detector systems developed and used for nuclear physics and nuclear data measurements with spallation neutron sources at the Los Alamos Neutron Science Center (LANSCE) at the Los Alamos National Laboratory.
\end{abstract}

International Workshop on Fast Neutron Detectors

University of Cape Town, South Africa

April 3-6, 2006

\footnotetext{
${ }^{*}$ Speaker
} 


\section{Introduction}

The term "Nuclear Data" refers to the body of knowledge of properties of atomic nuclei and their interactions with other nuclei and particles. For over half a century, these data have been shared through international agreements [1] because of their utility in applications ranging from fission and fusion energy to non-proliferation, non-destructive analysis and interrogation, astrophysics, geology and medicine, and for assessing the effects of natural radiation on electronics and humans in space and on earth. Neutron nuclear data constitute a very important part of these data because of the neutron's unique abilities to act as vector for energy production, to transfer ionizing energy to biological and electronic systems, to alter materials by displacing atoms, and to create radioactive isotopes which are signatures of elemental abundances or nucleosynthesis in distant stars. Simply put, neutron nuclear data comprise all nuclear reactions in which neutrons are present in either the intial or final state or both.

Neutron nuclear data are derived and improved by experimental measurements, nuclear model calculations, evaluations to provide complete data sets in a range of neutron energies, and tests of the data by integral exeperiments and neutron transport calculations to judge the quality of the evaluated data and calculational models. The improvement in the data over the past 30 years has been remarkable so that now one can calculate with some confidence the performance of systems as diverse as a conventional reactor or the effectiveness of shielding in an accelerator facility.

As these improvements continue, the demands on the accuracy of neutron nuclear data are also increasing. Because of economic limitations and also because calculations can be done, prototype nuclear reactors and critical test facilities might not be constructed in the future. Instead, new power reactors optimized for high burn-up of the fuel or for transmutation of radioactive waste will be designed solely on calculations. To specify the design margins, the uncertainties in the nuclear data must be known and included in the calculations. This problem becomes more challenging than just an uncertainty on a cross section at each energy; the covariances in the uncertainties must also be known.

I would like to emphasize in this review the system aspects of making experimental neutron nuclear data measurements, for the choice of a particular neutron detector is often influenced greatly by factors other than the sophistication of the detector material. Many measurements, and some of the highest quality, use detector materials that were developed more than fifty years ago. Significant advances in this field can often be attributed to other technologies, such as more intense neutron sources, better electronics, the continuing advances in computers that lead to better data acquisition, and greatly improved nuclear data themselves which are used in rapidly improving neutron transport codes that simulate physical processes so that the experimental data can be corrected and understood with greatly increased confidence.

This Workshop focuses on the development and application of neutron detectors. In this paper, I would like to give a brief overview of the field of neutron nuclear data and describe several neutron detector systems that are in use. The field is very large and is summarized every three years in international conferences that now go by the name of Nuclear Data for Science 
and Technology [2]. Hence, the selection of topics here will certainly not be comprehensive, and it will, of course, be biased by my personal experience at the Los Alamos Neutron Science Center (LANSCE) and other facilities. I apologize in advance for not including a very large number of advances that have been crucial to the field.

\section{Neutron Total Cross Sections and Neutron Detectors Systems}

The basic neutron interaction cross sections are based on total cross sections, for which measurements the efficiency of the neutron detector need not be known. Rather, the total cross section is determined by transmission "in good geometry" of neutrons through a sample that is well characterized in thickness, density, and composition. The term "good geometry" means that the neutrons that interact in the sample do not reach the neutron detector. Thus the sample is located far from the neutron source and the detector is significantly farther removed. An experimental program at Los Alamos produced total cross section data for materials from hydrogen to uranium with uncertainties often less than 1\% (Fig. 1) [3]. The detectors for these measurements were simple plastic scintillators. Only three detectors were used: a monitor scintillator in front of the sample to normalize the neutron fluence between target-in and targetout runs, and two BC404 plastic scintillators, $1.27 \mathrm{~cm}$ and $5.08 \mathrm{~cm}$ thick respectively, for neutron time-of-flight spectrometry. The energy dependent total cross section is then simply obtained from the ratio of sample-in to sample-out tof-spectra [Ref. 3].

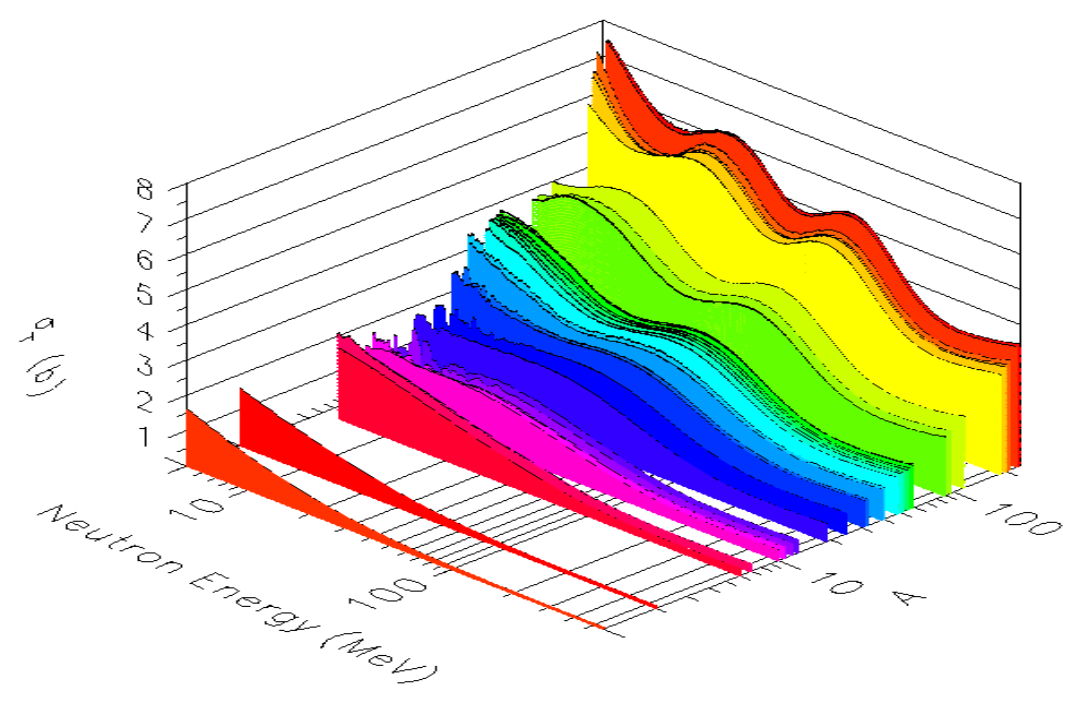

Fig. 1 -- Neutron total cross sections measured from 5 to $560 \mathrm{MeV}$ [3]. The overall uncertainties are typically $1 \%$ or better.

Although this measurement is simple in principle, the demands on the detectors are severe in order to achieve such small uncertainties. The pulsed, continuous-in-energy spallation source 
of fast neutrons at LANSCE [4] produces neutrons from over $600 \mathrm{MeV}$ down to less than 1 $\mathrm{MeV}$. The scintillator output is not uniform in time but rather reflects both the macrostructure of the neutron source (due to the duty cycle of the accelerator) and the structure in each micropulse. Possible instabilities of the photomultiplier gain, which can be caused by count rate variations during the experiment, were avoided by proper stabilisation and gain control.

A second systems aspect of this experiment results from the intensity of the neutrron source. An intense neutron source of course greatly facilitates obtaining results with low statistical uncertainties, but it also adds the possibility of pulse pileup or deadtime of the electronics and the data acquisition.

These important aspects of the total cross section measurement can be investigated by changing the intensity of the neutron source and by using samples of different thicknesses. In both cases, the results should not depend on the rate of neutrons detected.

\section{Neutron Detectors and Neutron Nuclear Data Standards}

The principal neutron cross section standard is $\mathrm{n}-\mathrm{H}$ differential elastic scattering. At energies in the $\mathrm{MeV}$ region but below $270 \mathrm{MeV}$, except for a negligible neutron capture reaction, the elastic scattering of neutrons by hydrogen comprises entirely the neutron total cross section. Thus one only needs to know the relative angular distribution of the elastic scattering in order to know differential cross section. One might think that, because the n-p reaction is so basic, it is well known either from experiment or from theory. Recent studies at $10 \mathrm{MeV}$ [5] and in the 160-196 MeV region [6] show that this is however not the case, that is, when one needs a standard that is not based on discrepant experimental data.

The n-H scattering standard is central to neutron detectors based on organic scintillators. For example, organic scintillators contain hydrogen and carbon and are often used as neutron detectors in the neutron energy range of $0.1 \mathrm{MeV}$ to several hundred $\mathrm{MeV}$. If the detector efficiency needs to be known, then, in many instances, it needs to be calculated using the n-H differential scattering cross section as well as data for neutron interactions with carbon. Cross section evaluations, based on the new experimental data, will result in some changes to the calculated detector efficiency. Although these changes can seem small, they will be important as we try to improve significantly the data base and to reduce uncertainties in it.

The $\mathrm{n}-\mathrm{H}$ scattering standard is also of particular importance in establishing other standards against which other cross sections are measured. For example, proton-recoil telescopes (PRT) use the n-H differential cross section particularly at backward center-of-mass scattering angles (forward-going proton recoils) for determining the flux of neutrons on a sample, such as an ion chamber containing a well characterized deposit of fissionable material. Fission cross sections can be determined in turn relative to the n-p differential cross section and then used to measure the neutron fluence in an experiment. We use parallel-plate fission ion chambers routinely as neutron flux monitors at all the neutron data beam lines at LANSCE [7]. They are rugged, last for years without changing the $\mathrm{Ar}-\mathrm{CO}_{2}$ fill gas, have good time response, require only a few electronic modules for signal processing, and are easily cross-compared. 


\section{New neutron detector systems, arrays and techniques - some selected topics}

\subsection{FIGARO - neutron-emission spectra via a double time-of-flight experiment}

One of the continuing challenges in measuring neutron emission from neutron-induced reactions is to obtain data comprehensively as a function of incident neutron energy. These data are of obvious importance in any neutron transport calculation. Two approaches are being developed for use with the continuous energy source of fast neutrons at LANSCE, one for neutron emission energies from $\sim 0.5 \mathrm{MeV}$ to $10 \mathrm{MeV}$, the other for emission energies from 50 to $400 \mathrm{MeV}$.

For neutron-emission energies below $10 \mathrm{MeV}$, the FIGARO array is under development at LANSCE [8]. This array consists of, at present, 20 EJ301 liquid scintillators [9] that provide pulse-shape discrimination above $\mathrm{En}=300 \mathrm{keV}$ and two gamma-ray detectors. We are measuring fission neutron spectra and multiplicities as a function of incident neutron energy over a range of incident neutron energies from $1 \mathrm{MeV}$ to $200 \mathrm{MeV}$ with first measurements on ${ }^{235} \mathrm{U}$ and ${ }^{238} \mathrm{U}[10]$.

The layout of the array is shown in Fig. 2. The neutron beam from the spallation neutron source is collimated to $1.3 \mathrm{~cm}$ in diameter. The time of flight of the incident neutrons indicates their energy. The energy spectra of fission neutrons are determined by time of flight over a 1meter flight path between the fission chamber and the neutron detectors. The fission signal serves as a start signal and the neutron detector as the stop signal. A fission chamber with spontaneously fissioning ${ }^{252} \mathrm{Cf}$ is used as the standard neutron spectrum against which the other measurements are referenced. The procedure described in detail in Ref. [8] to subtract background and to determine the neutron detection efficiency by measuring tof-spectra with a Cf-source can only be applied for measurements of similar neutron spectra at comparable conditions concerning spectral fluence and count rates. We have clearly observed the change in fission neutron spectra with the opening of so-called second-chance fission (n,n'f) and thirdchance fission (n,2nf). We have also quantified the fission spectrum and neutron multiplicity up to incident neutron energies of $200 \mathrm{MeV}$, data which are of importance in designing acceleratordriven systems for the incineration of nuclear waste and for energy amplifiers.

To measure the neutron-emission spectrum from a non-fissionable nucleus, gamma-ray detectors are used to provide the stop signal for time-of-flight of the incident neutrons from the source and also to provide the start signal for the time of flight of the neutrons emitted from the sample in the reaction. For these measurements, neutron elastic scattering of course cannot be detected. But most of the reactions populate excited nuclear states which decay by gamma-ray emission. We are working with theorists to develop nuclear reaction model codes that can calculate these "conditional" neutron spectra, that is, neutron emission that is correlated with the emission of a specific gamma ray in the residual nucleus. This gamma ray originates from a state with (usually) known spin and parity and thus restricts the spin distribution of states reached by neutron emission. More stringent tests of nuclear reaction models are expected to arise from the FIGARO data. 


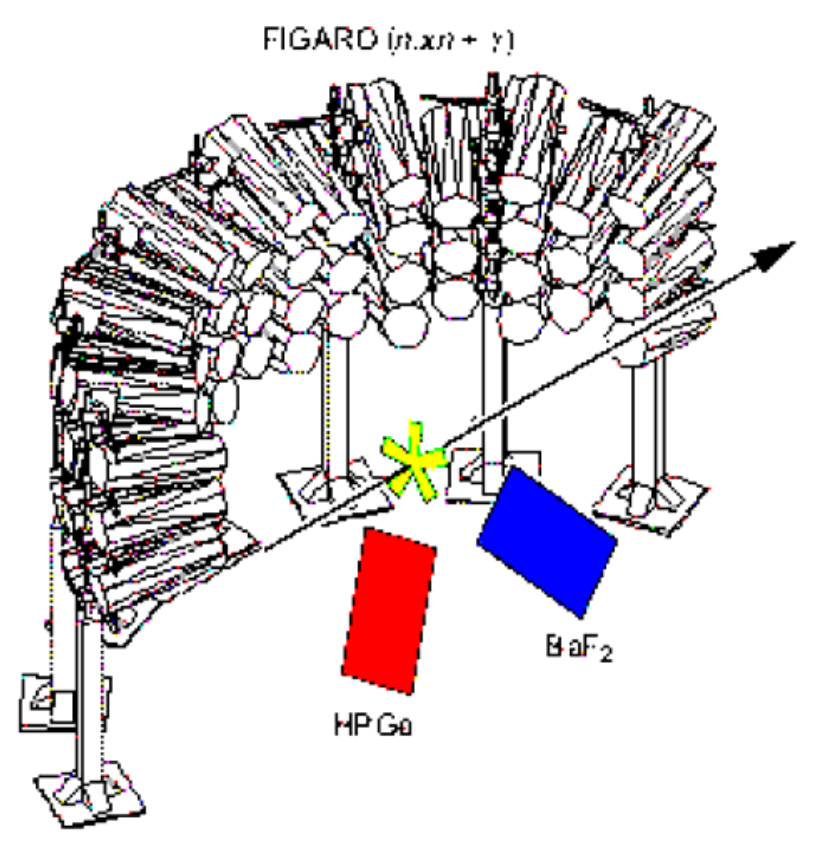

Fig. 2 - FIGARO array of neutron and gamma-ray detectors. The sample (or fission chamber) is placed at the "X", about 21 meters from the spallation neutron source.

\subsection{Spectroscopy of intermediate energy neutrons}

One of the major gaps in experimental measurements is of neutron emission, emission spectra and angular distributions from reactions induced by neutrons of intermediate energy, 50 $-400 \mathrm{MeV}$, that is double-differential $(\mathrm{n}, \mathrm{xn})$ measurements. Investigations have been made of $(p, x p),(p, x n)$ and $(n, x p)$ reactions, but $(n, x n)$ is more difficult because of a less intense neutron source and low efficiency detectors. It might be possible to calculate the $(\mathrm{n}, \mathrm{xn})$ neutron emission with models calibrated on the other three reactions, but this certainly needs to be verified.

An approach using a proton radiator and a novel type of proton recoil telescope is being carried out by a group from Kyushu University [11] at the white neutron source at LANSCE. The novel feature of their detector is a large $\mathrm{NaI}(\mathrm{Tl})$ to detect the recoil protons. At these energies, scattering and reactions of the $50-500 \mathrm{MeV}$ recoil protons in the $\mathrm{NaI}(\mathrm{Tl})$ is significant. To eliminate events where the proton or other charged-particle reaction products leave the $\mathrm{NaI}(\mathrm{Tl})$ before stopping, a plastic scintillator is placed on all of the side surfaces (Fig. 3 ). The detector then acts as a phoswich, and if there is a fast signal indicating energy deposit in the plastic scintillator, the event is vetoed. 


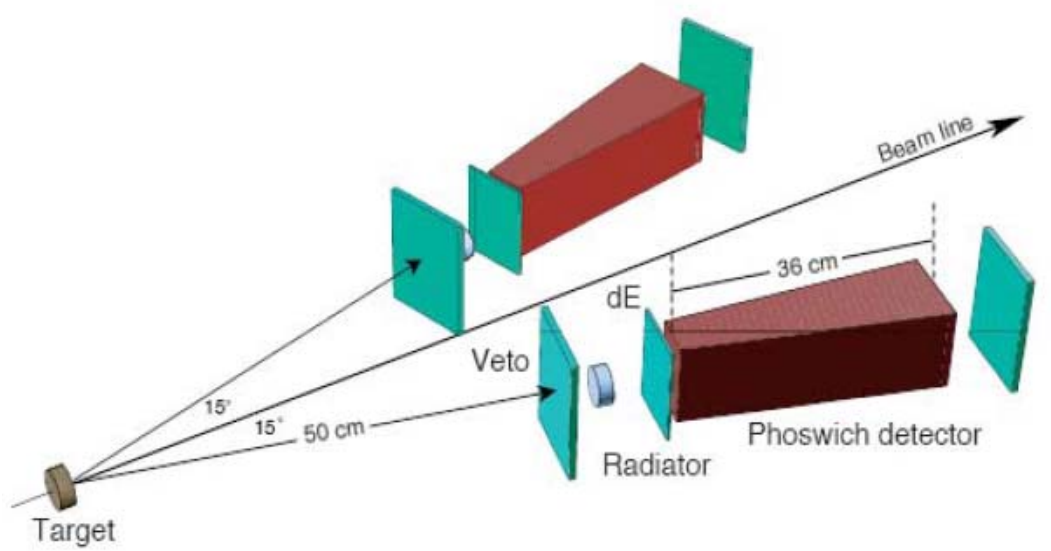

Fig. 3 - Detectors for neutrons up to $400 \mathrm{MeV}$ emitted from the target in (n,xn) reactions [11]. The $\mathrm{CH}_{2}$ radiator converts neutrons to proton recoils, which are then identified by the $\mathrm{dE}$ detector and stopped in the $\mathrm{NaI}(\mathrm{Tl})$ phoswich scintillator with a plastic scintillator veto counter on all the faces except that where the protons enter the detector.

\subsection{Elastic scattering with white neutron sources}

High accuracy total cross sections put a strict constraint on nuclear data evaluations in the sense that the partial cross sections must add up to the total. At many incident neutron energies, the elastic scattering cross section is a large fraction of the total cross section and must be known both for transport calculations and to constrain the non-elastic cross sections. At forward scattering angles, the differential cross section is large enough so that measurements could be made with the LANSCE white neutron source [12]. The detector consisted of a converter to make proton recoils, which were then tracked by wire chambers and scintillators and stopped in an array of CsI detectors. To obtain a larger range of scattering angles, a more intense source of neutrons is necessary and monoenergetic neutron sources are often the choice here [13].

\subsection{Fission neutron detectors in high flux environments}

Fission cross sections of ${ }^{235} \mathrm{U}$ and ${ }^{238} \mathrm{U}$ are considered to be standards for flux measurements and, as stated above, are used routinely at many facilities through parallel-plate fission chambers. In an environment of high radiation, such as produced by spallation neutron sources or electron-linac bremsstrahlung sources, the fast pulse of radiation can paralyze the detector and the electronics for some time before they recover and become active again. Compensated fission ion chambers [14] have been used to aleviate this problem by subtracting the effects of the radiation pulse on the fill gas and the structures in a dummy volume. At LANSCE we are developing a Lead Slowing-Down Spectrometer driven by $800-\mathrm{MeV}$ protons from the Proton Storage Ring. The radiation pulse here is much larger than that at conventional beam-target facilities and so the problems of detector paralysis are more severe. Compensated ion chambers will likely help, but our colleagues from the CEA (France) have also developed a compensation scheme for use with surface-barrier diodes, supplied as solar cells $[15,16]$. These 
compensated solar cells also have the advantage that a short-lived sample can be placed directly in front of the active cell for fission cross section measurements [17]. The flux normalization is accomplished by a second set of compensated solar cells with ${ }^{235} \mathrm{U}$ placed in front of one of them.

\subsection{Non-elastic scattering cross sections}

The measurement of neutron non-elastic cross sections is difficult and presents a challenge in the development of neutron detectors. Measurements with good accuracy would constrain the partial non-elastic reaction channels in data evaluations, and would also give a good value for the angle-integrated elastic scattering cross section, which is the difference between the total cross section (measured with excellent accuracy as discussed above) and the non-elastic cross section. In the neutron energy region above $30 \mathrm{MeV}$, neutron non-elastic cross sections are measured by transmission in "bad-geometry," by which is meant that the detector captures not only the transmitted neutrons but also those that are elastically scattered. The detector must be able to discriminate neutrons with the beam energy (perhaps modified somewhat if they scatter elastically from a light or medium weight nucleus) from neutrons inelastically scattered and from neutrons produced in reactions such as $(\mathrm{n}, \mathrm{xn})$.

At LANSCE, we have tried to make such measurements based on the approach used at a monoenergetic source [18]. Because of the inability to cleanly separate the elastically scattered neutrons from other neutrons, and because the detector covered only neutrons scattered in the forward direction ( $<45$ degrees), the resulting data had uncertainties of at best $5 \%$, similar to those of Ref. [18]. Improvements in neutron detectors and techniques remain a challenge for these measurements.

\section{Conclusions}

Neutron detectors continue to be essential to advances in the quality and the scope of neutron nuclear data. At the same time, nuclear data themselves are used in the design and the interpretation of results of detectors. A systems approach needs to be considered for the detectors, with consideration also of the performance of photomultipliers, high-voltage power supplies, electronics and data acquisition. New types of detectors and detector arrays are providing data for use in preparing evaluated data files and for benchmarking nuclear reaction codes used to interpolate within nuclear data sets and to extrapolate to new ranges of neutron energies and isotopes.

\section{Acknowledgments}

Most of the developments discussed here were accomplished by others at many laboratories. This presentation has benefited from support by the Los Alamos Neutron Science Center at the Los Alamos National Laboratory. This facility is funded by the US Department of Energy and operated by the University of California under Contract W-7405-ENG-36. 


\section{References}

[1] Nuclear data are available through several Internet web sites, such as http://www-nds.iaea.org

[2] International Conference on Nuclear Data for Science and Technology, Santa Fe, New Mexico, USA, September 26 - October 1, 2004, eds. R.C. Haight, M.B. Chadwick, T. Kawano, and P. Talou, AIP Conference Proceedings, vol. 769, Melville, NY, ISBN 0-7354-0254-X (2005).

[3] W. P. Abfalterer, F. B. Bateman, F. S. Dietrich, R. W. Finlay, R. C. Haight, and G. L. Morgan, "Measurement of Neutron Total Cross Sections up to $560 \mathrm{MeV}$," Phys. Rev. C 63, 044608 (2001). LA-UR-00-2215 (2000).

[4] P. W. Lisowski, C. D. Bowman, G. J. Russell, S. A. Wender, 'The Los Alamos National Laboratory Spallation Neutron Sources,” Nucl. Sci. Eng. 106, 208 (1990).

[5] N. Boukharouba, F. B. Bateman, C. E. Brient, A. D. Carlson, S. M. Grimes, R. C. Haight, T. N. Massey, and O. A. Wasson, "Measurement of the n-p Elastic Scattering Angular Distribution at En = 10 MeV," Phys. Rev. C65, 014004 (2001).

[6] S.E. Vigdor for the CE71 Collaboration, "Measurement of Absolute np Scattering Differential Cross Sections with a Tagged Intermediate-Energy Neutron Beam," Ref. [2] p. 820.

[7] S. A. Wender, S. Balestrini, A. Brown, R. C. Haight, C. M. Laymon, T. M. Lee, P. W. Lisowski, W. McCorkle, R. O. Nelson, W. Parker, and N. W. Hill, "A Fission Ionization Detector for Neutron Flux Measurements at a Spallation Source," Nucl. Instr. Meth. in Phys. Res. A336, 226 (1993).

[8] D. Rochman, R. C. Haight, J. M. O'Donnell, M. Devlin, T. Ethvignot, and T. Granier, "Neutroninduced Reaction Studies at FIGARO using a Spallation Neutron Source," Nucl. Instr. Meth. in Physics Research A523, 102 (2004).

[9] Eljen Technologies, Sweetwater, TX, USA.

[10] T. Ethvignot, M. Devlin, H. Duarte, T. Granier, R. C. Haight, B. Morillon, R. O. Nelson, J. M. O'Donnell, and D. Rochman, "Neutron Multiplicity in the Fission of 238U and 235U with Neutrons up to $200 \mathrm{MeV}$," Phys. Rev. Lett. 94, 052701 (2005).

[11] N. Shigyo, S. Kunieda, T. Watanabe, S. Noda, K. Ishibashi, Y. Iwamoto, D. Satoh, T. Nakamura and R. C. Haight, " Measurement of Continuous-Energy Neutron-Incident NeutronProduction Cross Section," Ref. [2] p. 924.

[12] J. H. Osborne, F. P. Brady, J. L. Romero, J. L. Ullmann, D. S. Sorenson, A. Ling, N. S. P. King, R. C. Haight, J. Rapaport, R. W. Finlay, E. Bauge, J. P. Delaroche and A. J. Koning, "Measurement of Neutron Elastic Scattering Cross Sections for ${ }^{12} \mathrm{C},{ }^{40} \mathrm{Ca}$, and ${ }^{208} \mathrm{~Pb}$ at Energies from 65 to $225 \mathrm{MeV}$," Phys. Rev. C70, 054613 (2004).

[13] J. Klug et al, "Elastic neutron scattering at $96 \mathrm{MeV}$ from 12C and 208Pb," Phys. Rev. C67, 031601(2003).

[14] P.E. Koehler, J.A. Harvey, and N.W. Hill, “Two detectors for (n,p) and $(n, \alpha)$ measurements at white neutron sources," Nucl. Instr. and Meth. A361 (1995) 270.

[15] T. Ethvignot, T. Granier, L. Giot, P. Casoli, R.O. Nelson, “A Fission-Fragment-Sensitive Target for X-ray Spectroscopy in Neutron-Induced Fission,” Nucl. Instr. Meth. in Phys. Res. A490, 559 (2002).

[16] M. Petit, T. Ethvignot, T. Granier, R.C. Haight, J.M. O’Donnell, D. Rochman, S.A. Wender, E.M. Bond, T.A. Bredeweg, D.J. Vieira, J.B. Wilhelmy, and Y. Danon, “A Compensated Fission Detector Based on Photovoltaic Cells," Nucl. Instr. Meth. Phys. Res. A 554, 340-346 (2005).

[17] D. Rochman, R. C. Haight, J. M. O'Donnell, A. Michaudon, S. A. Wender, D. J. Vieira, E. M. Bond, T. A. Bredeweg, A. Kronenberg, J. B. Wilhelmy, T. Ethvignot, T. Granier, M. Petit, and Y. Danon, "Characteristics of a Lead Slowing-Down Spectrometer Coupled to the LANSCE Accelerator," Nucl. Instr. Meth. in Phys. Res. A550, 397 (2005).

[18] C. Zanelli, P. Urone, J. Romero, F. P. Brady, M. Johnson, G. Needham, J. L. Ullmann, and D. Johnson, "Total Non-elastic Cross Sections of Neutrons on C, O, Ca, and Fe at 40.3 and 50.4 MeV,” Phys. Rev. C23, 1015 (1981). 